\title{
Molecular Dynamics Studies of the Ion Beam Induced Crystallization in Silicon
}

\author{
L.A. Marqués. \\ M.-J. Caturla: \\ H. Huang \\ T. Diaz de la Rubia
}

RECEMVE:

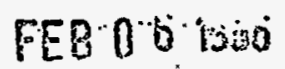

OSTI

This paper was prepared for submittal to the Materials Research Society 1995 Fall Meeting Boston, MA

November 27-December 2, 1995

January 23, 1995

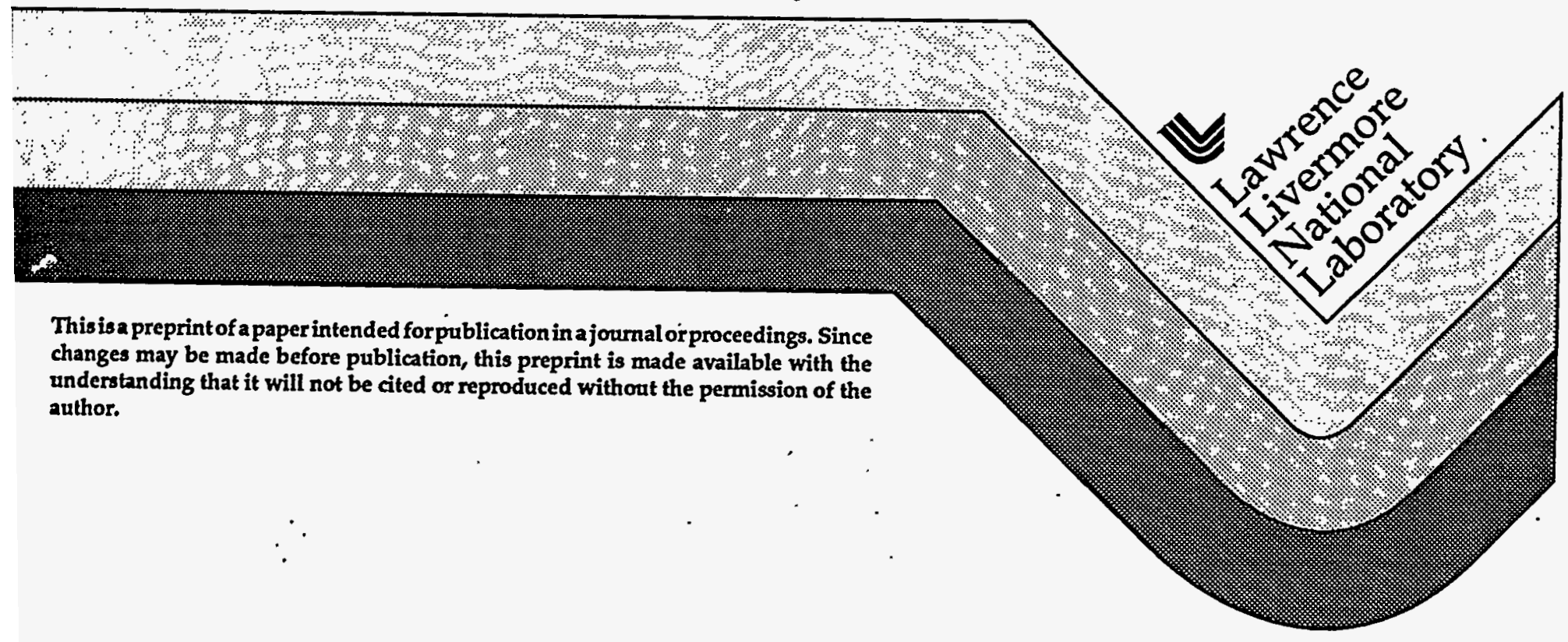

This is a preprint of a paper intended for publication in a journal or proceedings. Since changes may be made before publication, this preprint is made available with the understanding that it will not be cited or reproduced without the permission of the author. 


\section{DISCLAIMER}

This document was prepared as an account of rork sponsored by an agency of the United States Government. Neither the United States Government nor the University of Califormia nor any of their employees, makes any warranty, express or implied, or assumes anylegal liability or responsibility for the accuracy, completeness, or usefulness of any information, apparatus, produch, or process disclosed, or represents that its use would not infringeprivately owned rights. Reference herein to any specificcommercial products, process, or service by trade name, trademark, manufacturer, or otherwise, does not necessarily constitute or imply its endorsement, recommendation, or favoring by the United States Government or the University of California. The views and opinions of authors expressed herein do not necessarily state or reflect those of the United States Government or the University of California, and shall not be used for advertising or product endorsement purposes. 


\title{
MOLECULAR DYNAMICS STUDIES OF THE ION BEAM INDUCED CRYSTALLIZATION IN SILICON
}

\author{
L.A. MARQUÉS ${ }^{1}$, M.-J. CATURLA ${ }^{2}$, H. HUANG, T. DÍAZ DE LA RUBIA \\ Chemistry and Materials Science Department, L-268, Lawrence Livermore National \\ Laboratory, Livermore, CA 94551
}

\section{ABSTRACT}

We have studied the ion bombardment induced amoiphous-to-crystal transition in silicon using molecular dynamics techniques. The growth of small crystal seeds embedded in the amorphous phase has been monitored for several temperatures in order to get information on the effect of the thermal temperature increase introduced by the incoming ion. The role of ion-induced defects or the growth has been also studied.

\section{INTRODUCTION}

The amiorphous to crystal transformation in silicon is of considerable technological interest because of its potential applications in the field of microelectronic processing [1]. The crystallization of amorphous silicon starts with the nucleation of small.crystal clusters. Initially, these clusters are unstable because of the large surface-to-volume ratio and therefore tend to shrink. However, from thermodynamic considerations a few of them will become large enough for growth to be favoured. The nucleation and growth of small crystal clusters are thermally activated processes that have been described theoretically [2] and experimentally in silicon [3].

- Ion irradiation has been shown to dramatically enhance the solid phase crystallization of silicon with respect to the pure thermal process $[4,5]$. In particular, when amorphous silicon is bombarded by xenon ions at $1.5 \mathrm{MeV}$ the nucleation and growth rates can be enhanced by eight and four orders of magnitude, respectively, with respect to the pure thermal processes [4]. These experimental studies showed that the ion bombardment induced crystallization is controlled by beam parameters such as dose rate and average energy deposited in the solid by elastic collisions [5]. However, some of the aspects of the ion beam induced recrystallization are not yet fully understood.

While molecular dynamics (MD) simulation is a powerful tool that can be used to study. ion-induced dynamical processes at the atomic scale, the conditions of the experiment (doses of $10^{13} \mathrm{~cm}^{-2} \mathrm{~s}^{-1}$ and energies above $1.5 \mathrm{MeV}$ ) are far beyond the scope of the $\mathrm{MD}$ method. Nevertheless simple situations, such as defect-induced motion of a crystal-amorphous interface [6], can be simulated and some qualitative and quantitative data can be obtained. We have carried out molecular dynamics simulations in order to get some insight into the phenomenon of the growth of small crystal clusters embedded in an amorphous matrix when it is ion bombarded.

\section{MODEL *}

The most important feature in an MD simulation is the selection of the potential that describes the interactions between the atoms. We have used the Stillinger-Weber (SW) potential for silicon [7]. This potential is widely used since it is quite simple (a combination of two-body and three-body terms) and describes fairly well the properties of both crystal and

This work was performed under the auspices of the. U.S. Department of. Energy by Lawrence Livermore National Laboratory under contract No. W-7405-Eng-48. 
liquid phases of silicon. Of special interest in our study is the fact that the melting point of crystal silicon predicted by the potential $\left(T_{C L}=1691 \mathrm{~K}\right)$ is nearly the same as the value obtained experimentally.

The amorphous samples used in this study consist of 4096 and 32768 atoms. They were prepared by first melting $(8 \times 8 \times 8) a_{0}$ and $(16 \times 16 \times 16) a_{0}$ perfect crystal lattices, respectively, where $a_{0}$ is the equilibrium lattice parameter for silicon $\left(a_{0}=5.428 \AA\right)$. Afterwards, the two liquids were slowly cooled and the final amorphous structures were relaxed at $773 \mathrm{~K}$ $\left(500^{\circ} \mathrm{C}\right)$, since this is the temperature that has been used in the ion-induced crystallization experiments [5]. All the simulations were carried out at constant volume using periodic boundary conditions.

Since we are interested in the study of the growth behavior of crystal seeds in an amorphous matrix we inserted small crystalline clusters in the amorphous structures. In the case of the large sample we created a spherical hole in its center with a radius of $3.5 a_{0}$ where we inserted a perfect crystal grain with a radius of $3.3 a_{0}$. The sample was allowed to relax and equilibrate at $773 \mathrm{~K}$ for several picoseconds. We carried out the same procedure in the small sample creating a hole with a radius of $2.2 a_{0}$ and inserting a crystal grain with a radius of $2 a_{0}$. Since the holes are bigger than the inserted crystal grains, the total number of atoms is lower than in the pure amorphous samples: 32544 in the case of the big sample and 3982 in the case of the small one. This is not a problem since the crystal and amorphous silicon have different densities (the amorphous has $1 \%$ lower density than the crystal, both experimentally [8] and by simulation using the Stillinger-Weber potential [9]), and during the relaxation period the two structures evolve to match this density difference.

In order to monitor the growth of these small crystal seeds during our $\mathrm{MD}$ simulations it is necessary to use some procedure that allows the identification of the two phases in an effective way. Conventional criteria based on potential energy or coordination calculations are not effective in this case since the energetics and coordination of the atoms in the amorphous and crystalline phases are very similar [10]. We have observed that averaging the atom coordinates in the simulations for several femtoseconds the angular distribution of the crystal phase becomes narrower, due to the elimination of the thermal vibrations in the averaging process, while in the amorphous case it remains unchanged. This difference in behavior upon time averaging allows the introduction of a valid criterion to identify the amorphous and crystal phases of silicon. A more detailed description of this criterion is given elsewhere [11].

\section{RESULTS}

In order to compare to known experimental results we first annealed the 32544 atom sample for $350 \mathrm{ps}$ at $773 \mathrm{~K}$. No net grain growth was observed. This behavior is in agreement with the experiments on solid phase crystallization of amorphous silicon [3], where the obtained growth velocity at $773 \mathrm{~K}$ is lower than $1 \AA / \mathrm{min}$, a rate that is clearly out of range of feasible $M D$ simulations. However, as we shall see, the ion bombardment can affect the growth kinetics in such a way that is possible to monitor the process in the MD simulation time scale.

As it has been pointed out by Wang et al., the influence that ion bombardment has on crystal growth can be divided into thermal spike effects and damage induced effects [12]. The former are related to the heating of localized zones in the target, and the latter with those atoms that have been displaced from their initial positions and have potential energies above the ground state. These two effects could lead to either amorphization or crystallization of the target, but in our case we will be only interested in the conditions for the crystallization. 
The zones heated during the thermal spike regime, as it has been shown by molecular dynamics simulations [13], can be brought well above the melting point of crystal silicon or, in particular, above the melting point of amorphous silicon. The amorphous silicon described

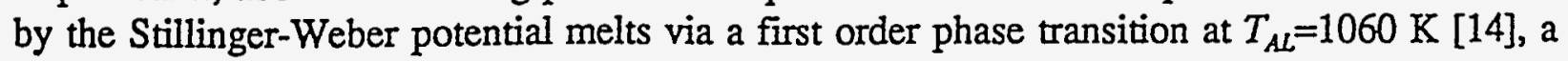
value $360 \mathrm{~K}$ below the experimental one [15].

We have observed that the growth rate of the crystal seeds increases with annealing temperature between $T_{A L}$ and $T_{C L}$. We prepared the 3982 atom sample at several temperatures $(1100 \mathrm{~K}, 1175 \mathrm{~K}, 1250 \mathrm{~K}, 1375 \mathrm{~K}$ and $1400 \mathrm{~K})$ by means of velocity rescaling and monitored the growth of the crystal grain during annealing for more than $600 \mathrm{ps}$. In the simulation we made rescaling of the velocities every picosecond in order to maintain a constant temperature. This is necessary since the energy released by the crystallization process accumulates increasing the temperature of the sample. This heat released can enhance the transformation of the nearby amorphous material producing an explosive crystallization [16].

For all these temperatures the crystal grain in the small sample is big enough to grow, i.e. its size is above the critical to show spontaneous growth. Initially, the grain grows linearly and maintains its spherical shape. Figure 1 shows the evolution of the grain radius with time for the different temperatures, as well as the best linear fits. The slope of these fits determine the growth rates $v_{G}$ which are also displayed in the Figure 1 . The values are somewhat smaller than the obtained in the simulation of laser induced epitaxial crystallization of silicon [17] probably due to the different interface geometry.

Close to the end of the simulations at temperatures of $1250 \mathrm{~K}$ and above, the grain adopted a cubic shape and the interfaces crystal-amorphous were (111) planes. This is a logic result since the epitaxial growth of the (111) face has the lowest growth rate, both experimentally [18] and by simulation using the SW potential [17]. The further growth from the (111) face frequently showed twining along the $<211>$ direction. In some cases we also observed crystallization in the hexagonal diamond structure, which is not surprising since the SW potential has the cutoff between the first and second neighbor shells and the energetics of the hexagonal and cubic diamond are the same [7].

The rates $v_{G}$ are represented in Figure 2 as an Arrhenius plot. We fitted these data to a function of the form:

$$
v_{G}=V_{G} \exp \left(-E_{G} / k_{B} T\right)
$$

where we obtained:

$$
\begin{aligned}
V_{G} & =1.409 \times 10^{4} \mathrm{~m} / \mathrm{s}, \text { and } \\
E_{G} & =1.03 \mathrm{eV}
\end{aligned}
$$

The classical theory of growth predicts that the growth velocity should be proportional to $v \exp \left(-\Delta G / k_{B} T\right)$, where $v$ is the rate at with atoms arrive to the amorphous-crystal interface and $\Delta G$ is the free energy barrier that an atom in the amorphous phase must overcome to transform to the crystalline phase [3]. $v$ is proportional to the diffusion coefficient $D$ in the amorphous phase which follows Arrhenius behavior:

$$
D=D_{0} \exp \left(-E_{D} / k_{B} T\right)
$$

Consequently, $E_{G}$ should be equal to $\Delta G+E_{D}$. We have carried out $\mathrm{MD}$ simulations to 


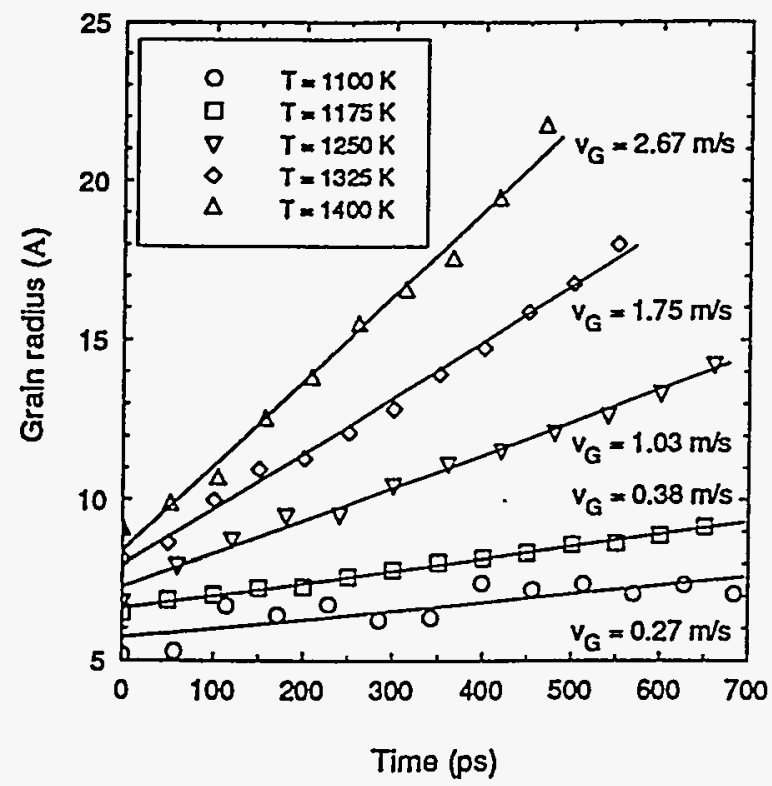

Figure 1. Evolution of the grain radius versus time for different temperatures. The solid lines are linear fits whose slopes define the growth rates $v_{G}$.

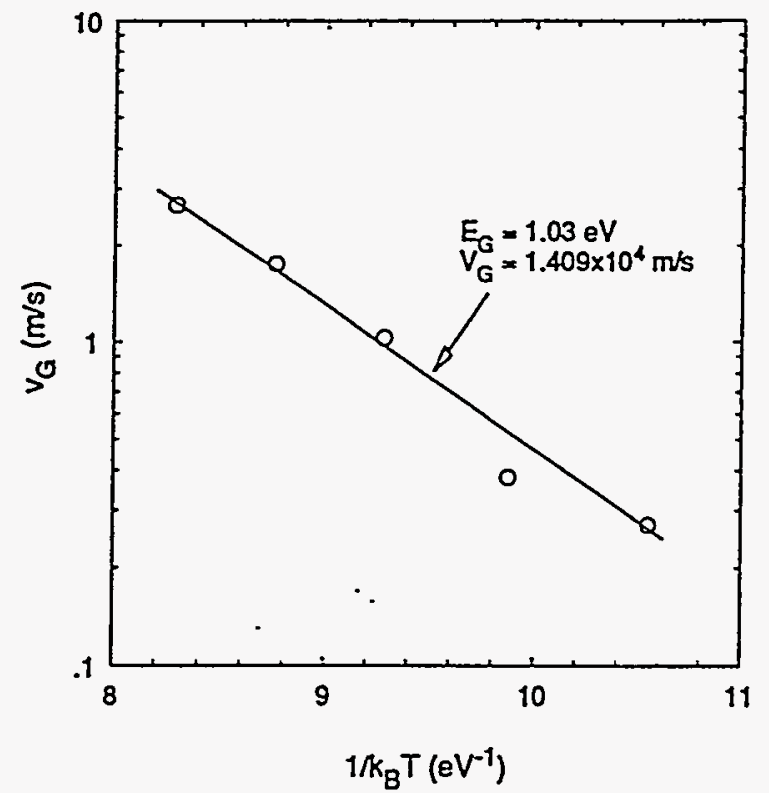

Figure 2. Arrenhius plot for the growth rates $v_{G}$. The solid line is the best fit to Eq. 1 .

determine the values of $E_{D}$ and $D_{0}$ between $T=770 \mathrm{~K}$ and $T=1700 \mathrm{~K}$. We monitored the mean square displacement for the atoms in the amorphous state for more than 1 ns. $D$ can be determined using the well known Einstein relation:

$$
D=\lim _{t \rightarrow \infty} \sum_{i=0}^{N} \frac{\left|\vec{r}_{i}(t)-\vec{r}_{i}(0)\right|^{2}}{6 N t}
$$

where $N$ is the number of atoms in the amorphous sample. The values obtained for $D$ are represented in Figure 3 as a function of temperature. The dashed lines are fits to an Arrhenius form, and the solid line shows previous results of Grabow et al. [17]. The discrepancy between our results and Grabow's can be explained if we take into account that their sample is a supercooled liquid and ours a superheated amorphous. Consequently, some difference in the atom mobility may be expected since the sample preparation, and possibly the average coordination, are not the same.

The value for $\Delta G$ is $0.34 \mathrm{eV}$ in the range of temperatures where we have studied the crystal growth. Below $T_{A L}$ the diffusion coefficients are of an order of magnitude lower. But the decrease in the atom mobility is not enough to justify the low growth rate obtained experimentally for temperatures around $800 \mathrm{~K}$. Consequently, below $T_{A L}$ the barrier $\Delta G$ should

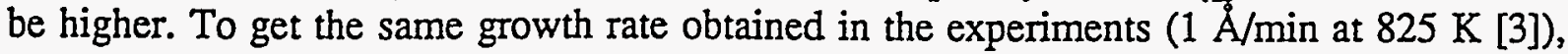
$\Delta G$ should be $1.7 \mathrm{eV}$, and thus $E_{G}=2.5 \mathrm{eV}$, which is not very far from the experimental value of $3.1 \mathrm{eV}[3]$.

\section{Induced damage effects}

In order to study the influence of ion-irradiation induced defects upon the growth behavior we put several low energy recoils in the amorphous matrix and monitored the evolution of the crystal grain size. We used the 32544 atom sample at $773 \mathrm{~K}$ with $20 \mathrm{eV}$ recoils initiated in the amorphous matrix. For this energy, the average range is approximately 


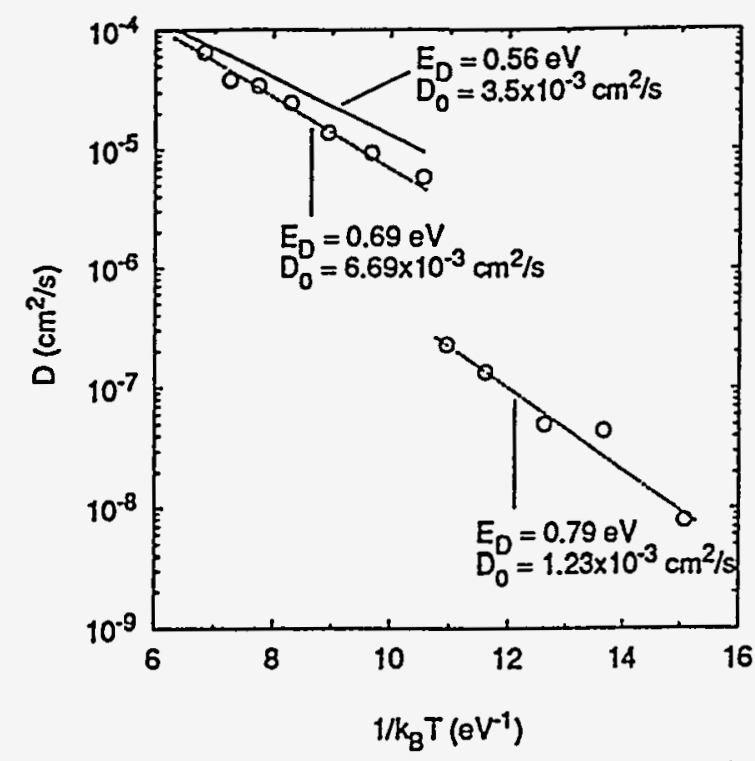

Figure 3. Arrhenius plot for the diffusion coefficients in amorphous silicon. Dotted lines are fits to Eq. 2 and the solid line shows previous results of Grabow et al. [17].

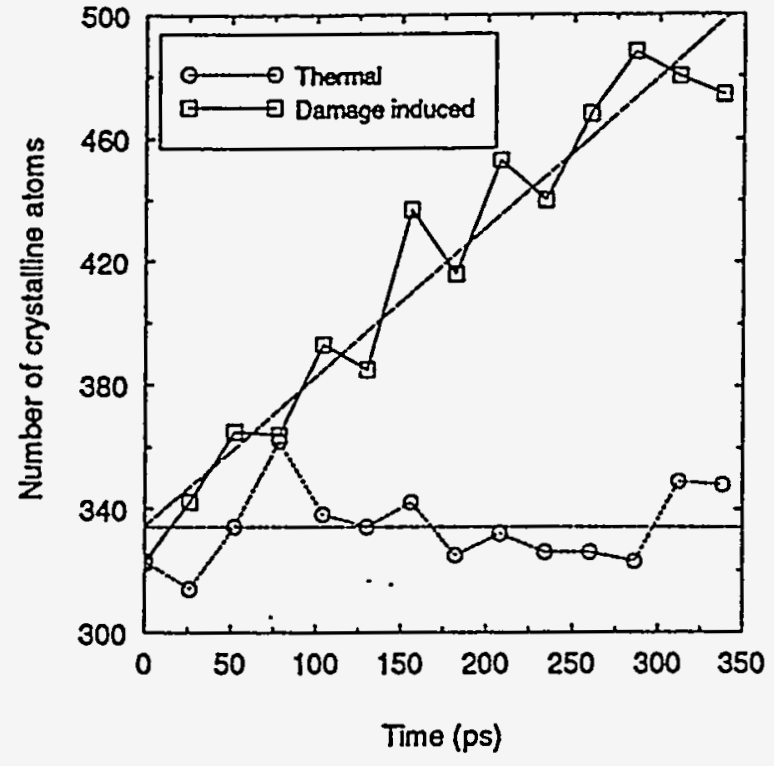

Figure 4. Evolution of the number of crystal atoms in the seed in for the thermal and damage induced cases.

$2.4 \AA$ and the probability of producing a permanent displacement (a defect) is $93 \%$ [6]. In the simulations we set 20 simultaneous recoils with their positions randomly chosen on a sphere with a radius of $3.5 a_{0}$. This radius is half a lattice unit bigger than the grain radius, approximately the range of the recoils. The velocities of the recoils were always directed towards the center of the crystal grain. The temperature of the atoms close to the cell boundaries were held fixed at $773 \mathrm{~K}$ using Brownian dynamics [19]. We followed the evolution of the system for $26 \mathrm{ps}$. This time is more than enough to dissipate the energy introduced by the recoils and reach again thermal equilibrium at $773 \mathrm{~K}$. In order to accumulate the effects of the recoils, the final configuration was the starting point for the next simulation. We kept constant the distance between the grain surface and the sphere where the recoils were started, as well as the ratio surface of the sphere/number of recoils in order to get a uniform rate in the grain growth. Therefore, as the grain grew we increased the number of recoils and the size of the sphere in the proportion mentioned.

The number of crystal atoms as a function of time is plotted in Figure 4. For the sake of comparison, we show as well the evolution in the pure thermal case. As it can be seen, there is no net growth in the thermal process, while in the case of the recoils growth is taking place. Clearly, at this temperature the injection of defects in the amorphous matrix near the crystal grain interface is necessary in order to produce noticeable growth. In other words, the mobility of the atoms in the amorphous phase at $773 \mathrm{~K}$ is too small to produce the reconstruction of the crystal lattice, but the recoils generate defects, i.e. atoms in a high potential energy state, that can easily anneal out by jumping to perfect crystal lattice positions at this low temperature when they are close to the crystal-amorphous interface. This is consistent with several experimental results where it is observed that the crystal grain growth rate is higher when the silicon is bombarded by heavy ions since they produce more defects [5], and with previous $M D$ studies of the defect-induced recrystallization of a planar crystalamorphous interface [6]. The slope of the curve gives a crystal growth rate of $0.5 \mathrm{~m} / \mathrm{s}$. 


\section{CONCLUSIONS}

We have simulated the ion beam induced amorphous-to-crystal transition in silicon. We took into account thermal spike and ion-induced damage effects, showing that in both cases the growth rate increases with respect to the pure thermal process. The growth of crystal seeds embedded in the amorphous matrix was characterized for several temperatures, and twining due to changes in the layer stacking during crystallization was observed. The same kind of twining has been observed in experiments on ion beam induced growth of preimplanted seeds in amorphous silicon [20]. This fact suggests that the local heating of the amorphous phase due the ion bombardment plays an important role in the microstructural evolution of small crystal seeds.

\section{REFERENCES}

1 Permanent address: Dept. de Electricidad y Electrónica, Facultad de Ciencias, Universidad de Valladolid, 47011 Valladolid, Spain.

${ }^{2}$ Permanent address: Dept. de Física Aplicada, Facultat de Ciencies, Universitat d'Alacant, Ap. 99, E-03080 Alacant, Spain.

1. I.W. Wu, A. Chiang, M. Fuse, L. Ovecoglu and T.Y. Huang, J. Appl. Phys. 65, 4036 (1989).

2. K.F. Kelton, A.L. Greer and C.V. Thompson, J. Chem. Phys. 79, 6261 (1983).

3. Y. Masaki, P.G. LeComber and A.G. Fitzgerald, J. Appl. Phys. 74, 129 (1993).

4. J.S. Im and H.A. Atwater, Nucl. Instr. and Meth. B 59, 422 (1991).

5. C. Spinella, A. Battaglia, F. Priolo and S.U. Campisano, Europhys. Lett. 16, 313 (1991).

6. M.J. Caturla, T. Díaz de la Rubia and G.H. Gilmer, J. Appl. Phys. 77, 3121 (1995).

7. F.H. Stillinger and T. Weber, Phys. Rev. B 31, 5262 (1985).

8. J.S. Custer, M.O. Thompson, D.C. Jacobson, J.M. Poate, S. Roorda, W.C. Sinke and F. Spaeden in Beam-Solid Interactions: Physical Phenomena, edited by J.A. Knapp, P. B фrgesen and R.A. Zuhr (Mater. Res. Soc. Proc. 157, Pittsburgh, PA, 1989) pp. 689-690.

9. W.D. Luedtke and U. Landman, Phys. Rev. B 37, 4656 (1988).

10. S.J. Cook and P. Clancy, Phys. Rev. B 46, 7686 (1993).

11. L.A. Marqués, M.-J. Caturla and T. Díaz de la Rubia, in preparation.

12. Z.L. Wang, N. Itoh, N. Matsunami and Q.T. Zhao, Nucl. Instr. and Meth. B 100, 493 (1995).

13. T. Díaz de la Rubia and G.H. Gilmer, Phys. Rev. Lett. 74, 2507 (1995).

14. W.D. Luedtke and U. Landman, Phys. Rev. B 40, 1164 (1989).

15. E.P. Donovan, F. Spaeden, D. Turnbull, J.M. Poate and D.C. Jacobson, J. Appl. Phys. 57, 1795 (1985).

16. W.C. Sinke, A. Polman, S. Roorda and P.A. Stolk, Appl. Surf. Sci. 43, 128 (1989).

17. M.H. Grabow, G.H. Gilmer and A.F. Bakker in Atomic Scale Calculations in Materials Science, edited by J. Tersoff, D. Vanderbilt and V. Vitek (Mater. Res. Soc. Proc. 141, Pittsburgh, PA, 1989) pp. 349-354.

18. E. Rimini, Ion Implantation: Basics to Device Fabrication (Kluwer Academic Publishers, Dordrech, 1995) p. 177.

19. D.W. Heermann, Computer Simulation Methods in Theoretical Physics, 2nd ed. (SpringerVerlag, Berlin, 1990), p. 55.

20. C. Spinella, S. Lombardo and S.U. Campisano, Appl. Phys. Lett. 57, 554 (1990). 